\title{
The Theoretical Study on Anomalous Experimental Phenomenon of Nd: Glass Pulsed Laser
}

\author{
Yingcai Wang, Lei Wang \\ Laser Institute, Hebei Academy of Sciences, Shijiazhuang, 050081, China
}

\begin{abstract}
Basing on the rate equations of the four-level laser system and a great deal of precise experimental data, and taking account of the influence of the overlooked spontaneous emission, we have theoretically studied the mechanism of the abnormal phenomena of light amplification without being pumped when the neodymium glass rod is elongated to exceed the length of the optical pump cavity. The result of theoretical study basically conforms to that of the experiment
\end{abstract}

Keywords: Abnormal experimental phenomenon Rate equation Spontaneous emission Light amplification

PACS: 42.55.-f, 78.45.+h, 42.62.-b

\section{INTRODUCTION}

In 1960, the first set of ruby solid laser was invented by T. H. Maiman in U.S. Hoghes Co., and in 1961, a neodymium glass solid laser was developed by E. Snitzer in American Optical Co. Since then, others had developed a variety of neodymium-doped crystal solid lasers such as Nd:YAG, Nd:YAP, NdPP, etc.

In most of common solid lasers a linear pumping lamp and an elliptical cylinder pump cavity are adopted. At that time, according to the basic principle of laser and the light gathering efficiency of the elliptical pump cavity, in both ruby solid laser and neodymium glass solid laser ( $\mathrm{Nd}$ :YAG, etc.) the pumping lamp and the laser bar were respectively placed on two focal lines in the elliptical cavity, making the pumping lamp, the laser rod and the elliptical cavity have equal lengen ${ }^{[1,2,3]}$ (the rod is hereinafter referred to as the equal-length rod, see the real line in figure 1). If not changing other conditions and only properly elongating the rod toward the outside of the pump cavity (the rod is hereinafter referred to as the elongated rod, see the dotted line in figure 1), the newly elongated parts of the working substance out of the pump cavity is unaffected by the optical pumping. Then according to the basic principle of laser, the pumping threshold of the elongated rod laser should rise and the pumping efficiency should fall $^{[7,9]}$.

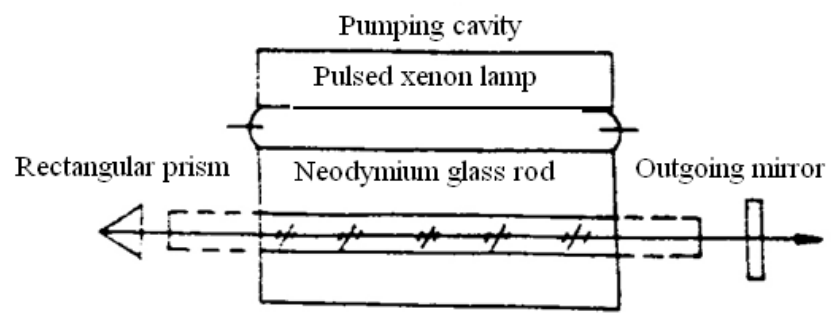

Figure1. Schematic diagram of experimental facility: Equal-length rod (real line); elongated rod (including the dotted line) 
But, in February 1972, our experiment of neodymium glass laser practically produced completely opposed result ${ }^{[5,6]}$, i.e. the elongated rod laser has the markedly lowered (rather than raised) pumping threshold and the markedly raised (rather than lowered) pumping efficiency. It is discovered by accident that the abnormal phenomenon of the extension rod light amplifications not consistent with the experimental facts。 Then, according to the fact in the experiment, we carefully analyzed the energy level's fine structure and the lasing procedure of $\mathrm{Nd}^{3+}$ ions in glass; and in the rate equation, we took into account the influence of the commonly overlooked spontaneous emission, conducting the theoretical studies on the mechanism of the abnormal experimental phenomenon of light amplification in the elongated $\operatorname{rod}^{[8,10]}$.

\section{The MAXimum Power OUtPut of Equal-Length Rod And Elongated Rod}

According to the rate equation of the four-level laser system, we get the maximum power output of the equal-length rod:

$$
p_{\max } \approx \frac{1}{4} h \vee \frac{c_{0}}{L+(n-1) l_{1}} T \Delta n_{t}\left(\frac{\Delta n_{0}}{\Delta n_{t}}-1\right)^{2}
$$

And the maximum power output of the elongated rod:

$$
p_{\max }^{\prime} \approx \frac{1}{4} h v \frac{c_{0}}{L+(n-1) l_{2}} T \Delta n_{t}\left(\frac{\Delta n_{0}}{\Delta n_{t}}-1\right)^{2}
$$

where $h v$ is the photon energy, co is the velocity of light in a vacuum, $n$ is the dielectric refractive index, $T$ is the transmissibility of the outgoing mirror, $L$ is the cavity length of the resonator, $l_{1}$ is the length of the equal-length rod and $l_{2}$ is the length of the elongated rod; $\Delta n_{1}=\left(n_{3}-n_{2}\right)_{t}, \Delta n_{0}=n_{3}-n_{2}$. (1) and (2) show that the maximum power output is in inverse proportion to the dielectric length in the resonator. By reason that $l_{2}>l_{1}$, the following formula should be established:

$p_{\max }^{\prime}<p_{\max }$

But, practically, our experimental result was contrary to this formula, i.e. $P_{\max }^{\prime}>P_{\max }$. This is the abnormal experimental phenomenon of light amplification in the elongated rod.

\section{Theoretical Studies}

After the abnormal experimental phenomenon of light amplification in an elongated rod was published, our colleagues have made comprehensively experimental verification and theoretical approach to this phenomenon. Some colleagues thought that the scattering of the working substance (rod) forms certain pump power density in the parts of the rod out of the pumping lamp, leading to a decrease in pumping threshold and an increase in pumping efficiency; however, the theoretical viewpoint was unable to clear up this phenomenon. It is because Figure 2 (the photo of the equal-length neodymium glass rod and the elongated neodymium glass rod) markedly indicated that the effect of ultraviolet radiation of optical pumping, on the elongated rod, only that portion that overlaps the equal-length rod is colored, and the newly elongated parts out of the pump cavity is unaffected by ultraviolet radiation and not colored. However, the photographs of laser output spot comparison in Figure 3 and Figure 4 showed that the elongated rod has a much stronger laser output intensity and a more uniform power-density distribution of light beam cross section as compared with the equal-length rod. Then, what is the mechanism of producing the abnormal experimental phenomenon? This is a theoretical problem deserving of earnest study. 


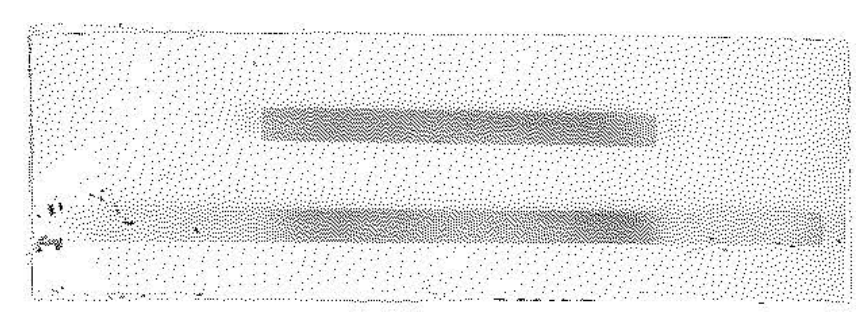

Figure2. Comparison of ultraviolet radiation coloring of optical pumping on the equal-length rod (upper) and the elongated rod (lower)

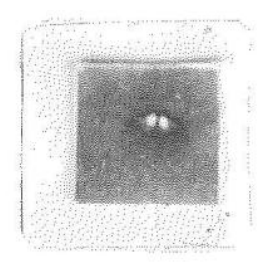

(a)

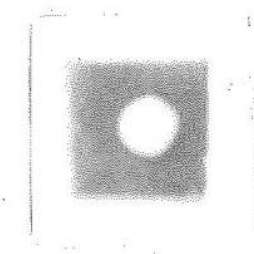

(b)

Figure3. Q-Switch pulse (or Dynamic) output spot comparison of equal-length rod (a) and the elongated rod (b) with the same input (80J)

\subsection{Basic Train of Though}

Based on the law of energy conservation, we carefully analyzed the experimental material, method and result, and thought that this phenomenon is caused by the overlooked spontaneous emission in the rate equation of the four-level laser system.

In the course of solving the maximum power output of the equal-length rod, one considered only the inverted population density, $\triangle n_{0}$, at the pulse build-up starting time (i.e. $t=t_{0}$ ), and overlooked the previous accumulative process of $\triangle n_{0}$. In the three-level laser system, this method of handling problem perhaps is justifiable; however, in the four-level system it is not necessarily true. This is because $E_{3} \rightarrow E_{2}$ transitional inversion in the four-level system may take place at a very small pumping rate and doesn't need a high pumping rate necessary to keep population balance in the three-level system.

Generally speaking, a high pumping rate is often used in the giant pulse four-level laser system. When the pumping rate reaches a certain value and the population realizes high reversion, the amplification of spontaneous emission takes place. In this way, before forming giant pulse laser, the spontaneous emission near the end face of the working substance (rod) can produce very strong luminous flux, as a result, most or all population in the upper laser level is used up. When population reaches the upper laser level within $0 \sim t_{0}$ of time, part of population of $E_{3}$ in the upper level spontaneously falls on the low level, and the decrement rate of number varies directly with the population in the upper level.

$\frac{d n_{3}}{d t}=-A_{32} n_{3}$

Where, $A_{32}$ is the spontaneous transition probability and its dimension is $S^{-1}$. It gives the probability at which the particles in $E_{3}$ energy level spontaneously fall on $E_{2}$ energy level.

Spontaneous emission is a space-time statistic function. For a large number of particles of spontaneous emission, there is no phase relation among different emission processes, emissive photons are irrelevant. Their characteristics may be represented by the particle age in the high-energy state. When exceeding this age, the particles spontaneously return to the low level and produce radiant energy; the stronger the spontaneous emission is, the more the energy loss becomes. Of course, this is 
a condition that we do not want. To inhibit spontaneous emission and to decrease energy loss are our basic train of thought in the study of the mechanism of the abnormal experimental phenomenon of light amplification in the elongated rod.

\subsection{Raising the Utilization Ratio of Inverted Population}

The solution of equation (4) is

$n_{3}\left(t_{0}\right)=n_{3}(0) \exp \left(\frac{-t_{0}}{\tau_{32}}\right)$

where, $n_{3}\left(t_{0}\right)$ is the particle number of energy level $E_{3}$ loss before the laser giant pulse is formed, $\tau_{32}$ is the spontaneous emission lifetime of $E_{3}$.

Suppose that only those spontaneous emission photons which form a very small angle with the rod axis and pass through the whole length of the rod can produce the markedly amplified spontaneous emission, and the amplified spontaneous emission produces a strong beam from the two ends of the rod. Its solid angle approximates as:

$\Omega \approx \frac{n^{2} A}{l^{2}}$

Where, $n$ is the dielectric refractive index, $l$ and $A$ are the length and the sectional area of the rod respectively. Obviously, when $l=L, \Omega$ is minimal, i.e. $\Omega_{\min }$. In this way, such a slender rod has a longer light path under the action of high gain it can establish stimulated emission in various non-coherent spontaneous decays. However, in practical situations (for example, equal-length rod), often $l_{1}<L$; in this way, only the population of spontaneous transition shown in the following formula can establish stimulated emission

$n_{3}^{\prime}\left(t_{0}\right)=n_{3}(0) \exp \left(\frac{-t_{0}}{\tau_{32}}\right)\left(\frac{\Omega_{\min }}{\Omega}\right)$

When the laser giant pulse forms (i.e. $t=t_{0}$ ), the population participating in laser oscillations in the upper laser level is:

$n_{3}^{\prime \prime}\left(t_{0}\right)=n_{3}(0)\left\{1+\exp \left(\frac{-t_{0}}{\tau_{32}}\right)\left[\frac{\Omega_{\min }}{\Omega}-1\right]\right\}$

By reason that

$\frac{\Omega_{\min }}{\Omega}=\frac{l^{2}}{L^{2}}$

the substitution of (9) into (8), leads to:

$n_{3}^{\prime \prime}\left(t_{0}\right)=n_{3}(0)\left\{1+\exp \left(\frac{-t_{0}}{\tau_{32}}\right)\left[\frac{l^{2}}{L^{2}}-1\right]\right\}$

Where, the second term in the braces on the right side expresses the loss caused by spontaneous emission. Owing to $l_{2}>l_{1}$, the elongated rod has less loss caused by the spontaneous emission as compared with the equal-length rod. In this way, the utilization ratio of population participating in laser oscillations in the upper laser level is raised. This point had been confirmed by the difference between the equal-length and elongated rods in our experiment. 


\subsection{The Explanation on the Abnormal Experimental Phenomenon of Light Amplification}

Let the elongated rod in Figure 1 be a cylindrical rod with a diameter of $D_{2}\left(D_{2}=D_{1}\right)$ and a length of $l_{2}\left(l_{2}>l_{1}\right)$, the total active population be $N^{\prime}$. Because the elongated and equal-length rods received optical pumping of the same volume, $N^{\prime}=N$. According to (10), the elongated rod has less loss caused by spontaneous emission as compared with the equal-length rod, therefore $n_{3}^{\prime}>n_{3}$. And because $n^{\prime}{ }_{2}$ $=n_{2}=0$, it is apparently that

$\Delta n_{0}^{\prime}>\Delta n_{0}$

$\Delta n_{t}^{\prime}=\Delta n_{t}$

$\frac{\Delta n_{0}^{\prime}}{\Delta n_{t}^{\prime}}>\frac{\Delta n_{0}}{\Delta n_{t}}$

substituting (12) into ( 2) gives

$p_{\max }^{\prime} \approx \frac{1}{4} h v \frac{c_{0}}{L+(n-1) l_{2}} T \Delta n_{t}^{\prime}\left(\frac{\Delta n_{0}^{\prime}}{\Delta n_{t}^{\prime}}-1\right)^{2}$

comparing (13) and ( 1) gives

$p_{\max }^{\prime}>p_{\max }$

\subsection{Amendment of (13)}

(13) is derived upon the condition that the absorption loss of the working substance is neglected; in fact, this loss cannot be neglected. If we consider the absorption loss of the working substance, replace $l_{2}$ in (13) with $L$, let $l=L$ and regard $l$ as a variable, (13) may be changed into

$p_{\max }^{\prime} \approx \frac{1}{4} h v \frac{c_{0}}{n l} T \Delta n_{t}^{\prime}\left(\frac{\Delta n_{0}^{\prime}}{\Delta n_{t}^{\prime}}-1\right)^{2}$

where, $\Delta n_{t}^{\prime}=\frac{1}{B_{s} \tau_{c}^{\prime}} ; \frac{1}{\tau_{c}^{\prime}}=\frac{1}{\tau_{c}}+\frac{1}{\tau_{l}} ; \tau_{l}=l\left(1-e^{-\mathrm{K}_{\mathrm{o}} l}\right) c^{-1} . \kappa_{\mathrm{o}}$ is the absorption coefficient per unit length of the cylindrical rod, $l$ is the length of both the optical resonator and the rod.

By using (15) we can qualitatively analyze the law in which $P^{\prime}{ }_{\text {max }}$ change with $l$ under the condition

of the same pumping rate. On the basis of the equal-length rod, $\Delta n_{0}^{\prime}$ and $\Delta n_{1}^{\prime}$ increase and $\frac{c_{0}}{n l}$

decreases with the elongation of $l$. Because there is a square relationship between $P_{\text {max }}^{\prime}$ and $\frac{\Delta n_{0}^{\prime}}{\Delta n_{t}^{\prime}}$, all

we have to discuss is the varied value of $\frac{\Delta n_{0}^{\prime}}{\Delta n_{t}^{\prime}}$ with the elongation of $l$.

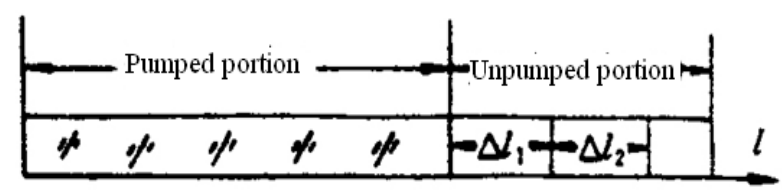

Figure4. Schematic diagram of the pumped and un-pumped portions of an elongated rod 
1)In certain range of elongated $l$, for example, the portion of $\Delta l_{1}$ in Figure 4 , as 1 elongates, $\frac{\Delta n_{0}^{\prime}}{\Delta n_{t}^{\prime}}$ increases; therefore $P^{\prime}{ }_{\text {max }}$ in (15) increases, the threshold lowers and when $\frac{\Delta n_{0}^{\prime}}{\Delta n_{t}^{\prime}}$ is maximal, $P^{\prime}{ }_{\text {max }}$ is also maximal, and the threshold is minimal. At this moment, the length of $l$ is the best length under the condition of the same pumping rate, let it be $l_{j i a}$. In this case, the single-pass gain obtained by the elongated section is more than its single-pass absorption loss.

2)If $l$ is elongated to $l>l_{\mathrm{jia}}, \frac{\Delta n_{0}^{\prime}}{\Delta n_{t}^{\prime}}$ decreases; therefore, $P_{\text {max }}^{\prime}$ decreases and the threshold increases. In this case, the single-pass gain obtained by the section of $l-l_{j i a}$ is less than its single pass loss.

On the contrary, if $l$ is shortened, $\frac{\Delta n_{0}^{\prime}}{\Delta n_{t}^{\prime}}$ decreases. When $\frac{\Delta n_{0}^{\prime}}{\Delta n_{t}^{\prime}}=1$, the generation and loss of laser have the same velocity, leading to a situation without laser output, i.e. $P^{\prime}{ }_{\max }=0$. The length of the rod, $l$, at this moment is called the cut-off length of the rod, $l_{j i e}$, without laser output.

To sum up the above analysis, we have more or less known that the length of the rod begins with $l=l_{j i e}$, as $l$ elongates, the Q-switch pulse (or dynamic laser power output) is an approximate curve with an ascending exponential form and the pumping threshold is a approximate curve with a descending exponential form. When $l=l_{j i a}$, the dynamic outputs are maximal, and the threshold is minimal. At this time, because the decreased solid angle of light beam from the end face of the rod, the light spot of dynamic outputs become smaller. When $l>l_{j i a}$, the dynamic output is a approximate curve with a descending exponential form and the threshold is a approximate curve with an ascending exponential form. As $\Delta n_{0}^{\prime}$ increases, $\Delta n_{t}^{\prime}$ also increases. For the dynamic curve, the descending velocity is slower than the ascending velocity; and for the dynamic threshold, the ascending velocity is slower than the descending velocity.

In conclusion, under the condition of the same pumping rate, if the length of the rod $l=l_{j i a}$, the dynamic output efficiency will be maximal and the dynamic threshold will be minimal. At this time, the beam divergence angle becomes smaller as compared with that at $l<l_{j i a}$. Furthermore, as the loss of spontaneous emission decreases, the storage time of population shortens and the laser buildup time becomes faster, therefore, when Q-switching, single pulse is easily obtained. This point has been confirmed in laser range- finding ${ }^{[4]}$.

\subsection{Method and Result of Experimental Verification}

In order to get accurate and reliable result of verification, in the experimental verification, a neodymium glass rod was gradually cut short. The result of dynamic verification is seen in Figure 5.

Of course, when raising an objection to the classical laser principle, we are prudent. It is necessary to obtain a great deal of experimental data; therefore, the experiment has been repeated many times.

It is It is seen from Figure 5 that when $l_{j i a} \approx 1.5 l_{\text {deng }}$, the dynamic output power has been increased by almost 3 time, and the pumping threshold has been reduced by about $34 \%$ as compared with the dynamic output power in the equal-length rod. Figure 4 showed that at this time the whole light spot of laser output is smaller and the power-density distribution is more uniform than that of the equal-length rod. 


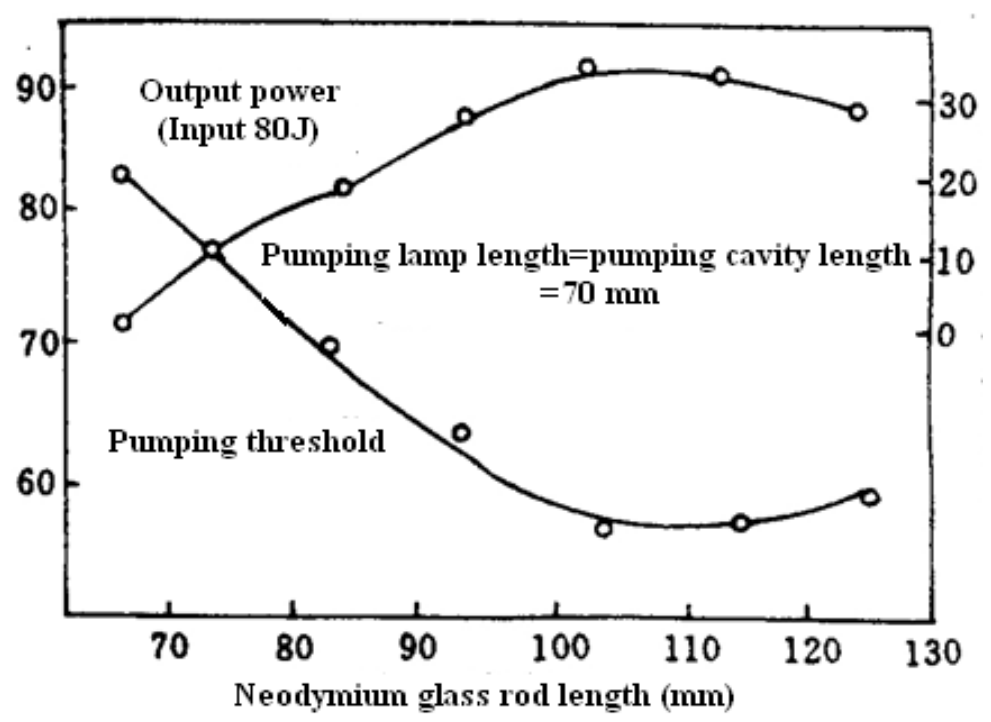

Figure5. Curve of $Q$-Switch(or dynamic )pumping threshold and output change in relation to rod length

From Figures 5 we are easy to determine the cut-off rod length under the condition of the same pumping rate and without laser output; in this experiment, $l_{j i e} \approx 65 \mathrm{~mm}$. Furthermore, we also adopted a medium-sized laser with an elliptical cavity length of $l_{t w o}=200 \mathrm{~mm}$ to do the experimental verification, getting similar verification results. Of course, experiments with large-sized lasers will also get similar verification results.

The aforementioned experimental verification result basically conforms to our theoretical analysis result.

\section{Conclusions}

Achievement of experimental study on this abnormal experimental phenomenon has been used and popularized in a certain type of home-made laser range finder, obtaining an obvious benefit. If it is used in the large power neodymium glass laser system researching laser fusion, the benefit will certainly be very obvious too.

The theoretical researching achievement of the abnormal experimental phenomenon has a very high academic and reference value in the applied research of solid laser used in four-level system, the applied and basic research of physical change process of laser formation in solid laser used in fourlevel system, and the fundamental research of interaction of light and substance.

\section{REFERENCES}

[1] compiled-translated by Tianjin University, Laser and Technologypp (Science Press, Beijing, 1972)

[2] W. Koechner, Solid-State Laser Engineering (NewYork, Spriger,1976) pp:319

[3] Compiled by Huazhong Institute of Technology Laser Technology et al(Hunan Science \& Technology Press, Hunan,1981)pp:44

[4] S.W Zhang, Y.C wang, Q-switch Laser Ranging of Low Speed Instant Turning Motor(News in brief). Laser,8 (11): 64(1981)

[5] Y.C wang,Chinese Physics Laser,13(6); 433 435 (1986)

[6] Y.C wang, Experimental Study of Threshold and Efficiency with Elongof Laser, 16(1):10 5(1995)

[7] H.zhang et al Pump Energy Distribution in Rod-shaped Amplifier Calculated by Using Monte Carlo Method, by Using Monte Carlo Method, Acta OpticaSinica, 17(2):1061 1067(1997) 
[8] Y.C wang, L.wang Mechanism of Light Amplification Phenomenon in Pulsed Neodymium Glass Laser with Elongated Rod, Progress of Laser and Optoelectronics,12:1924 (2001)

[9] J.B Chen, .Laser principle and opplicatio( Electronis Indusry Press,Beijing.2007)pp102

[10] Y.C wang,at al The theoretical study on abnormal exoerimental phenomenon of the elongated rod Nd:glass puled laser ,Acla physica sinica (2012)pp:19 ,1942070-1

\section{AUTHOR's BIOGRAPHY}

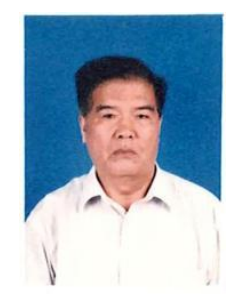

WangYingcai, (1938-) male, Senior engineer, mainly engaged in Extended rod Ndglass laser Basic research on the application of such aspects. 